\title{
Capitolo 9. Appunti lessicali sul Misogallo romano (n. 407)
}

\author{
Cristelli, Stefano
}

\begin{abstract}
The paper deals with some lexical issues raised by one of the Romanesco poems (n. 407) included in the so-called Misogallo romano (late 18th century). In particular, the work tries to explain four rather puzzling words: 1) pilacche, which possibly has to be considered a copying mistake for pilucche 'wigs'; 2) Mambrucche (in the syntagma aria de Mambrucche), to be linked to the French song Malbrough s'en va-t-en guerre; 3) tricche tracche, whose meaning could be just, as usually in Romanesco, 'a kind of instrument used in the Holy Week'; 4) policche (in the phrase fà policche), still obscure, for which it is nonetheless possible - among other proposals - to establish a comparison with similar words occurring in the dialects of Todi and Subiaco.
\end{abstract}

DOI: https://doi.org/10.1515/9783110677492-009

Posted at the Zurich Open Repository and Archive, University of Zurich

ZORA URL: https://doi.org/10.5167/uzh-195873

Book Section

Published Version

The following work is licensed under a Publisher License.

Originally published at:

Cristelli, Stefano (2020). Capitolo 9. Appunti lessicali sul Misogallo romano (n. 407). In: Faraoni, Vincenzo; Loporcaro, Michele. «E parole de Roma»: Studi di etimologia e lessicologia romanesche. Berlin: De Gruyter, 139-162.

DOI: https://doi.org/10.1515/9783110677492-009 


\title{
Stefano Cristelli
}

\section{Capitolo 9}

\section{Appunti lessicali sul Misogallo romano (n. 407)}

\begin{abstract}
The paper deals with some lexical issues raised by one of the Romanesco poems (n. 407) included in the so-called Misogallo romano (late 18th century). In particular, the work tries to explain four rather puzzling words: 1) pilacche, which possibly has to be considered a copying mistake for pilucche 'wigs'; 2) Mambrucche (in the syntagma aria de Mambrucche), to be linked to the French song Malbrough s'en va-t-en guerre; 3) tricche tracche, whose meaning could be just, as usually in Romanesco, 'a kind of instrument used in the Holy Week'; 4) policche (in the phrase fà policche), still obscure, for which it is nonetheless possible - among other proposals - to establish a comparison with similar words occurring in the dialects of Todi and Subiaco.
\end{abstract}

\section{Sangue de Bio! appoggerem panacche: problemi d'interpretazione}

Il testo 407 del Misogallo romano recita: ${ }^{1}$

1 In calce al sonetto è riprodotto l'apparato di Formica/Lorenzetti (1999, 489); per una scheda su questo prezioso lavoro cf. D'Achille (2009), dove si raccolgono e discutono anche le puntua-

Nota: Il lavoro nasce in seno al progetto di ricerca Grammatica storica del romanesco [SNF 100012_169814/1, 2018-2021]; ringrazio Vincenzo Faraoni e Michele Loporcaro per gli utili consigli ricevuti in fase di stesura. Sono altresì grato a Giulio Vaccaro di avermi permesso di accedere ai dati offerti dal corpus ATR (Archivio della Tradizione del Romanesco, su cui cf. Vaccaro 2012, 80). I brani della Cronica di Anonimo romano e dei Sonetti di Belli sono citati rispettivamente da Porta (1979) e Belli (1998); si dà solo il riferimento interno al testo (capitolo o numero di sonetto; rigo o verso), omettendo la pagina dell'edizione (il numero del verso, là dove segua direttamente il testo, è inserito senza altra indicazione tra parentesi tonde). Le glosse alle forme registrate dai vocabolari sono tendenzialmente fedeli alla fonte; in qualche caso è sembrato utile operare dei piccoli interventi. È impiegata la lettera $V$ per rappresentare, entro una successione di foni, una vocale qualsiasi.

Stefano Cristelli, Università di Zurigo

https://doi.org/10.1515/9783110677492-009 
«Sangue de Bio! appoggerem panacche

Su sti Frosci razzaccia de’ Berlicche

Sonaje cor martino quattro ntacche

Là 'n de le Trippe de' peccati ricche.

Gammeri! che finente er tricche tracche

Vonno arrubà, e vonno fà policche

De quanto c'è de bono, e empj le sacche.

V'infilzeremo l'ova colle picche,

Con un par de serciate alle Pilacche

Senza ce repricà ne acche, o ocche

Roscie je vojo fa le bianche gnucche

$E$ je vojo sonà certe sajocche:

Faje cantà su l'aria de Mambrucche:

Qui de’ Nizza non son le genti alocche.

1: bell'appoggià panacche $Y$. 3: intacche $Y$. 4: 'n telle trippe $Y$. 5: siente $Y$ (prob. lapsus per sinente 'persino'); tricche tricche $B$, tricche tracche $Y$. 6: arubane $Y$; pelicche $Y$. 7: empì $Y$. 8: Dove ponno trovà le genti micche $Y$. 9: pilucche $Y$. 10: Senza replica fà d'un acche un'ocche $Y$. 12: certe bajocche $Y$. 14: non sò $Y$; genti alocche $B Y$, genti sciocche $B^{2}$ ».

Nel complesso, il senso del componimento è chiaro. L'autore esordisce annunciando una serie di violenze a danno dei Francesi (sprezzantemente nominati, come di consueto nel Misogallo romano, Frosci), ${ }^{2}$ diavolacci (razzaccia de' Berlicche) contro cui si augura di veder indirizzati anzitutto percosse (panacche) e colpi di coltello (martino). Dopo le accuse infamanti dei versi 4-7, che dipingono la gente d'Oltralpe come parassitaria e rapace (incerta, stando al commento, l'interpretazione di tricche tracche e fà policche), il testo si fa ancor più minaccioso: gli stranieri saranno castrati (V'infilzeremo l'ova) per mezzo di picche e raggiunti da sassate (serciate; incerto il significato di Pilacche); le loro bianche gnucche (da intendere 'nuche, teste') si dovranno tingere, per le ferite, di rosso; inoltre a tanta violenza, si specifica, non sarà possibile replicare (ne acche, o ocche vale 'né ai né bai'). In chiusura, il poeta ribadisce la volontà di lapidare i rivali (je vojo sonà certe sajocche) e termina il sonetto con il desiderio

lizzazioni esposte in Marucci (2001); sul Misogallo romano cf. inoltre Lorenzetti (1999a) e Lorenzetti (2004), di cui pure si ha notizia nel lavoro di D'Achille. Il sonetto è tràdito da due testimoni: B (Roma, Museo Centrale del Risorgimento, ms. 221, testo di base) e Y (Città del Vaticano, Biblioteca Apostolica Vaticana, ms. Ferrajoli 719), per la cui descrizione si rimanda a Lorenzetti (1999b, rispettivamente 192 e 196-197). Le maiuscole rispecchiano quelle degli originali (cf. Lorenzetti 1999b, 191).

$2 \mathrm{Su}$ questo aspetto cf. Formica $(1999,81)$. Per le forme citate nelle prossime righe (escluse, naturalmente, quelle che hanno motivato il presente intervento) bastino il commento e il glossario dell'edizione (entrambi a cura di L. Lorenzetti); non si riportano le varianti di Y, delle quali, almeno in riferimento alle voci più oscure, si farà adeguata menzione più avanti. 
che gli stessi siano costretti a cantare su un'aria ben definita (quella - non identificata nel commento - di Mambrucche) l'umiliante affermazione secondo cui 'in Italia la gente non è allocca' (così se, come sembra, Qui de’ Nizza va letto 'di qua da Nizza').

Se il sonetto risulta globalmente intellegibile, nel medesimo si annidano, come si è avuto modo di anticipare, quattro voci per cui il pur denso e puntuale commento di L. Lorenzetti non offre riscontri o glosse tali da fugare ogni dubbio: si tratta delle forme in rima tricche tracche, policche, pilacche (d'ora in avanti la parola sarà scritta con la minuscola) e Mambrucche (o meglio del sintagma aria de Mambrucche). I paragrafi che seguono sono dedicati a queste voci: ${ }^{3}$ dopo aver riferito le osservazioni di Lorenzetti, si raccoglieranno tutti i dati utili a commentare la parola in esame e si proverà, dove possibile, a illustrarne il significato; il riferimento alle alternative offerte dalla tradizione sarà frequente e, nel caso di pilacche (pilucche nel ms. Y), occuperà una parte considerevole del discorso. Gli strumenti messi a punto dalla lessicografia romanesca (e laziale) degli ultimi vent'anni giocheranno talora un ruolo decisivo; ${ }^{4}$ più in genere, permetteranno di inquadrare la circolazione di alcune forme con una quota di raffronti significativa.

Si tratterà per primo il caso di pilacche (§2), poi quelli di Mambrucche (§3) e tricche tracche (§4); ci si soffermerà al termine del lavoro (§5) su policche, la cui interpretazione pone diversi problemi.

\section{Pilacche o pilucche?}

Nelle note di commento al sonetto, in corrispondenza della forma pilacche si legge: «Pilacche (pilucche) parrebbe 'teste', ma senza riscontri»; del termine si dà notizia anche nella Nota linguistica che precede la raccolta, dove tra le «novità» lessicali offerte dal Misogallo viene incluso per l'appunto il pilacche del testo 407,

3 È intenzionalmente trascurata gammeri, parola «di difficile interpretazione» nel contesto secondo il glossario allestito da Lorenzetti (s.v. gammero): per spiegarla potrebbe essere sufficiente ipotizzare una deformazione eufemistica di cancheri, voce interiettiva attestata tanto in italiano quanto nel dialetto di Roma (in Belli: cf. VBel, s.v. cànchero; si tratterebbe insomma di un caso simile - non identico, perché gammeri 'gamberi' era parola realmente esistente - a quello di sangue de Bio al verso 1).

4 Il commento ai sonetti misogallici non poteva ancora avvalersi, per es., dell'utilissimo corpus ATR (cf. la nota d'apertura); è poi il caso di ricordare che dal 1999 a oggi, oltre a risorse informatiche di ottimo livello, ma d'indirizzo più generico (un nome per tutti: Google Libri), sono stati prodotti nuovi repertori del romanesco (si pensi a Malizia, Di Nino 2008 e Vaccaro 2010) e delle odierne varietà laziali (qualche esempio: PNor, VCC, VCor, VDBl, VDBr). 
parafrasato però, ivi, con "'palle, testicoli (?)'» (Lorenzetti 1999b, 157, in nota). Rilevante anche ciò che si afferma nel glossario dell'edizione (s.v. pilacche): «s.f. ignoto alla documentazione rom., senso proprio e origine oscuri: dal contesto [...] parrebbe deform. gerg. (in rima) per teste» (glossa confluita in Di Nino 2008, che registra la forma misogallica s.v. pilaccia). Dunque pilacche, parola altrimenti sconosciuta al romanesco, potrebbe indicare i 'testicoli' o - ma non è ben chiaro il rapporto tra le due glosse - le 'teste' dei Francesi. Un'ulteriore verifica nei repertori di Roma e del Lazio non dà risultati migliori: pilacche non trova riscontri. ${ }^{5}$

Proviamo a mettere ordine. Scorrendo il sonetto ci si accorge sùbito che, lasciando a testo pilacche, la struttura rimica risulta piuttosto insolita: ABABABAB ACDCDC. ${ }^{6}$ La lezione testimoniata dal ms. Y (cf. il §1) dà meno difficoltà; leggendo pilucche si ha infatti uno schema molto più comune: ABABABAB CDCDCD. ${ }^{7}$ Lorenzetti, come si è visto poc'anzi, ipotizza per pilacche una deformazione in rima: se s'interpreta bene, però, il riferimento dello studioso non è all'alterazione di pilucche in pilacche nel ms. $\mathrm{B}$, bensì alla struttura apparentemente gergale della voce (tanto nella variante in -acche quanto in quella in -ucche), della cui forma la rima in -Vcche sarebbe, in definitiva, responsabile. In altre parole, non sembra che nell'edizione si faccia riferimento all'incongruenza strutturale del ms. di base o, comunque, alla necessità di valorizzare pilucche: le attenzioni si fissano sulla sola forma in -acche, che non è mai indicata come una possibile, banale corruzione di pilucche, ${ }^{8}$ ed è glossata in modo

5 Non ha ovviamente valore il confronto con il cal. pilacca 'pilacchera' (cf. NDDC, s.v.); andrà scartato anche il sic. pilacca 'stivaletto femminile; giacca lunga di panno pesante' (cf. VS, s.v.), che pure darebbe un qualche senso nel contesto del sonetto.

6 Lo schema sarebbe ancor più strano (ABABBBAB ACDCDC) se si seguisse da vicino il ms. di base e non si accogliesse l'intervento dell'editore al verso 5 , dove a tricche tricche di $\mathrm{B}$ è preferito tricche tracche di Y (cf. il §1).

7 Sulle principali forme del sonetto in Italia cf. Beltrami (2011, 239-247), che alla p. 247 cita un componimento caproniano con schema ABABABAB ACDCDC proprio per illustrarne l'irregolarità (calcolata); si aggiunga che la sirma su due rime alternate (CDCDCD) è fra i tratti caratteristici del sonetto sei-settecentesco (cf. Magro/Soldani 2017, 112, 128 e 139-140) ed è quasi esclusiva, infatti, nelle Povesie in lengua romanesca di Micheli (cf. Costa 1999, 70-72; in nessun caso ACDCDC).

8 Vista una delle glosse proposte nel commento, vale a dire 'teste', e visti gli esempi di 'pilucca' 'testa pelata' che si citeranno più avanti, si potrebbe pensare che si sia vista in pilacche per l'appunto una deformazione di pilucche 'teste pelate': la forma pilacche, però, è definita nel glossario di «senso proprio e origine oscuri» (cf. supra), senza riferimento a 'pilucca" e al suo significato. Inoltre, se davvero pilucche fosse stata riconosciuta come forma poziore, la variante di Y sarebbe stata trasferita a testo: in Lorenzetti (1999b, 189) si informa che il lavoro di edizione è stato condotto «mantenendo il più possibile integra la lezione del manoscritto che si è portato di volta in volta a testo e correggendo tale lezione, col ricorso alle altre, solo nel 
ambiguo (da un lato 'palle, testicoli'; dall'altro 'teste'). Un'indagine sulla diffusione di 'pilucca` nei dialetti italoromanzi, nonché nella storia del romanesco, può offrire invece altri ottimi argomenti - non ultimo quello semantico - per preferire la lezione di Y; fuori dell'ottica testuale, dà l'occasione d'illustrare con profitto un tipo trascurato nel commento al sonetto.

Stando ai dati esposti nel DIDE (s.v.), pilucca, pe- è termine noto ad alcune varietà italoromanze (corso, toscano [lucchese], siciliano, calabrese e sardo; per l'abruzzese è attestato pëlucchë) nei significati di 'parrucca, zazzera', 'rimprovero' e ‘sbornia' (accezione assegnata ai soli calabrese e siciliano gergali; per questo valore e per 'rimprovero' cf. anche Prati 1940 e PDG, s.v.); quanto all'etimologia, Marcato osserva che «Potrebbe trattarsi di riflessi diretti dello spagnolo e catalano peluca 'parrucca' (come sostiene il DEI)» oppure di voci dipendenti «dall'italiano parrucca, perrucca (probabile francesismo, DELI) con accostamento paretimologico a pilus, pelo. Per [...] il significato di 'sbornia' [.. .] si potrebbe risalire allo spagnolo peluca 'ubriachezza'». Alla testimonianza del DIDE si può accostare quella del LEI (vol. 4, 1567-1568), che cita forme simili

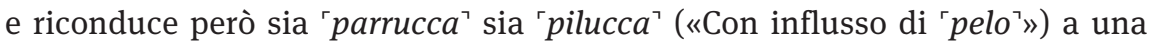
base * par(r)-/* per(r)-. Andrà aggiunto che pilucca 'parrucca' è attestato anche nel VDSp (s.v., accanto a piluccóne 'parruccone'); la variante con $e$ protonica è documentata per Viterbo da DIVVI, LDVit (anche 'zazzera' e accanto a peluccone 'zazzeruto') ${ }^{9}$ e VVit (s.v.). Ancora: il VCVo (s.v.) attesta pelukka 'pelurie'; nel Folignate (a Foligno, a Trevi e di nuovo a Spello) e in Val di Pierle, oltre che in un isolato centro del Viterbese (Fabrica di Roma), le forme pilucca, pe- compaiono con il significato di 'testa pelata' rispettivamente secondo VTF (s.v. pilukka), ${ }^{10}$ VVPi (s.v. pilùkka) e VDFR (s.v. pelùkka), mentre il solo VCort (s.v. pilùcca) attesta il significato di 'taglio dei capelli, in genere': per questi ultimi esempi si deve forse pensare a derivati di *PǏLŪCCĀRE ${ }^{11}$ (cf. REWS, 6506, dove pilucca 'testa pelata' è registrato per il dialetto di Cortona).

Lasciando da parte i rapporti che legano le forme e i significati succitati, ciò che importa notare in questa sede è che pilucche, variante del ms. Y, è una

caso di incongruenze di rima o di palesi problemi di comprensibilità» (e in effetti è secondo questo principio che tricche tracche di Y sostituisce tricche tricche di B).

9 Peluccó, peluccóne 'zazzeruto, con i capelli folti e prolissi' è anche a Civita Castellana (cf. VCC, s.v.).

10 La forma vale anche 'qualità dell'erba'; pilucca 'filo d'erba' è nel todino (cf. VTT, s.v.). Il VTF (s.v.) registra a Trevi la variante pirukka.

11 L'esistenza di questa base è messa in dubbio dal DELI (s.v. piluccàre), che nota come «La spiegazione tradizionale [...], che ipotizza un lat. parl. *piluccāre, der. di pilāre 'pelare' non rend[a] ragione del suffisso»; ora l'EVLI (s.v. piluccàre) ripropone *PǏLūCCĀRE e trae il verbo da *P̌LUUCCU(M), dim. di PľLUS. 
lezione accettabile sul piano semantico non meno che su quello strutturale: sia che s'interpreti con 'parrucche', sia che lo si faccia con 'teste pelate', il brano dà senso. A fare la vera differenza è, nel nostro caso, un indizio interno alla storia letteraria del romanesco. Anche se a Roma la documentazione attesta quasi solo le forme per(r)ucca e par(r)ucca (nelle accezioni di 'parrucca'12 e di 'sbornia'), ${ }^{13}$ infatti, vi è un brano de La Libbertà Romana acquistata e defesa di Micheli (XI, 92-93; cf. Incarbone Giornetti 1991, 258) - siamo dunque pochi decenni prima del Misogallo - che offre un importante termine di paragone rispetto al pilucche di Y:

«Quel ${ }^{14}$ ce va: ie 'l dimanna e l'orbo allora, credenno che sia quel de le zeccate, senza responne' (taffe) te ie ammolla col su' bastone 'na terribbil zolla,

Che al mozzorecchio cusì ben azzecca, giusto giusto, in tel mezzo de la gnucca, che 'l fongo abbasso ie fa annà', de zecca, e a scirocco ie manna la pilucca».

12 Anche figuratamente, a indicare il sesso femminile: un esempio nel sonetto 560 di Belli ( $L a$ madre de le Sante, al verso 10).

13 Cf. Chiappini (s.v. perucca 'sbornia') con le relative Aggiunte e postille di Rolandi (s.v., dove si integra il significato di 'parrucca') e con il commento di Belloni/Nilsson-Ehle (1957, s.v. perùcca 'sbornia'; la parola sarebbe intanto uscita d'uso), VBel (s.v. perucca 'parrucca', che registra anche gli alterati perucchino e peruccóne; cf. anche, s.vv., perucchière 'parrucchiere' e peruccóne 'vecchione'), Ravaro (s.v. perùcca 'parrucca; sbornia'; documentati anche, s.vv., perucchière 'parrucchiere' e peruccóne 'persona anziana, noiosa, pedante') e Vaccaro (2010, s.v. perùcca 'sbornia'); la consultazione dell'ATR conferma la netta preponderanza del tipo con vibrante (nei testi del corpus il significato della voce è sempre quello di 'parrucca'; un'unica, parziale eccezione è rappresentata dall'occorrenza belliana di cui si è detto alla nota precedente). In effetti, di pilucca 'parrucca' dà conto, tra i repertori, solo Di Nino (2008, s.v. perucca), che cita l'occorrenza micheliana di cui si parlerà a breve (unico esempio del tipo nell'ATR); per quel che riguarda il pilucca presente nella RVRM (s.v., allato a perucca), la prudenza impone di non attribuire la forma al romanesco: l'attestazione, che non è commentata da Merlo, potrebbe appartenere ad un'altra varietà e andare a ingrossare, quindi, la serie di occorrenze citate poco fa a testo. È bene specificare infine che il pelucche presente in uno dei sonetti di Giacomo Palmiro Bompadre (Li capelli de la donna) è solo all'apparenza interessante; uno sguardo al contesto è sufficiente per capire che l'occorrenza in questione non fa al caso nostro: «Mo che so' escite fòra 'ste parucche / bisogna stacce attenta fiji belli / specie co' 'ste donnette un po' bacucche. / Dateje 'na tirata a li capelli, / si v'arimane in mano quer «pelucches / mannatela a fa' 'n giro a li Castelli!» (9-14; cf. Bompadre 1978, 25).

14 Il pronome si riferisce al mozzorecchio ('avvocato') protagonista, con il cliente che aveva a lungo derubato, della seconda scenetta raccontata da Clelia nel canto XI: l'azzeccagarbugli, spinto con una trappola a chiedere l'ora a un cieco, riceve da quest'ultimo una sonora bastonata. 
Qui pilucca vale evidentemente 'parrucca'; ed è d'altronde lo stesso autore, nelle note di commento al poema, a glossare pilucca con perucca. Quanto alla rima con gnuсca, condivisa dal sonetto misogallico, vale la pena di osservare che già nel Meo Patacca (VII, 77, 7-8) s'incontra gnucca : perucca: «Gl'azzolla una scopata in su la gnucca, / E te gl'attacca foco alla perucca» (Rossetti 1966, 257). ${ }^{15}$

Vero è che i rivoluzionari si distinguevano per il rifiuto della parrucca e più in genere della moda d'ancien régime (cf. ad es. Giorgetti 1993, 248-255); si potrebbe almeno ricordare, però, che «Gli stessi Marat, Danton e Robespierre continua[ro]no, secondo la tradizione settecentesca, a mettere in mostra sulle orecchie, ridotti a uno per parte, i larghi buccoli imbiancati di poudre» (Di Monaco 1999, 241); inoltre il sonetto - che in effetti si rivolge genericamente ai Frosci $-{ }^{16}$ può ben alludere a un accessorio identificativo della moda d'Oltralpe, a prescindere dalla sorte delle capigliature francesi sullo scorcio del sec. XVIII. ${ }^{17}$

Insomma: le pilucche di Y saranno le parrucche incipriate dei Francesi (di qui il sintagma bianche gnucche), non le loro teste pelate, come pure si potrebbe

15 Si ha invece perucca : zucca nel sonetto 265 di Belli (L'editto pe tutto l'anno): «Sortanto ho 'nteso un quèquero in perucca / a bbarbottà, svortannose de fianco: / «Chi cce governa, nun tiè ssale in zucca»» (9-11).

16 È il caso di notare che a Roma, dopo l'assassinio di Bassville (1793), l'ostilità antifrancese raggiunse un livello tale che "persino gli ecclesiastici emigrati - e dunque fedeli al papa - [iniziarono] a essere perseguitati», mentre il «popolo [. . . ] invocava che tutti i Francesi presenti a Roma e nel Paese venissero cacciati con la forza (o, secondo alcune ricostruzioni, addirittura uccisi [...])»(Formica 1999, 83-84). Già alla fine del 1792 l'ambasciatore sardo a Roma, C. D. Priocca, aveva notato «un certo astio quasi naturale, che [il popolo romano aveva] contro i Francesi, accresciuto dal fondo di religione, ch'egli conserva[va] in mezzo ai vizi ed alla ferocia» (cf. Formica 1999, 83, in nota).

17 Si può aggiungere, inoltre, un elemento che riguarda più da vicino Bassville, figura centrale per la genesi del Misogallo romano: in Révolutions de Paris del 2-9 febbraio 1793 (n. 187), in un'acquaforte che ritrae l'assassinio del diplomatico (l'immagine è posta tra le pp. 290 e 291), la vittima sembra indossare proprio una parrucca (riproduzione digitale del numero all'indirizzo https://gal lica.bnf.fr/ark:/12148/bpt6k10513568/f1.item); di un pezzo molto simile, più tardo ma a colori, si ha notizia dalla pagina http://parismuseescollections.paris.fr/fr/musee-carnavalet/oeuvres/13janvier-1793-23-nivose-an-ir-de-la-repque-assassinat-de-basseville-agent\#infos-principales [ultimo accesso alle due fonti: 21.3.2019]. Visti anche i dati raccolti alla nota 16, sembra interessante riportare quel che si legge nel suddetto numero di Révolutions de Paris, p. 292, a proposito della posizione politica di Bassville: «aujourd'hui il n’y a pas de milieu; il faut être ou aristocrate émigré, ou patriote ardent. Le modérantisme ne peut servir à rien aux yeux des puissances étrangères, qui ne peuvent plus, dans leur rage, distinguer ces nuances. Bassville n'étoit certainement pas un patriote. Nommé par le ministère constitutionnel, c'étoit un de ces hommes qui aiment à nager entre deux eaux; il se plaisoit à déclamer contre le peuple français; il nous trouvoit féroces; il a appris à ses propres dépens que les nations régies par un despote le sont encore davantage». 
interpretare sulla scorta di alcuni dialetti moderni (cf. supra); ed è proprio in questo senso che si può offrire una prima integrazione al commento di Lorenzetti: è possibile evidenziare, cioè, che il testo 407 - almeno nella forma documentata da uno dei mss. latori - offre una preziosa occorrenza di pilucca 'parrucca', voce di cui si ha traccia non solo in altre parlate italoromanze, ma anche in un noto autore della letteratura in romanesco. Alla luce dei dati raccolti più sopra, per converso, sembra azzardato attribuire al pilacche di B lo status di novità lessicale; si potrebbe trattare persino di una parola-fantasma. ${ }^{18}$

\section{Malbrough s'en va-t-en guerre, o l'aria de Mambrucche}

Quanto all'aria de Mambrucche, Lorenzetti osserva: «non è chiaro a quale opera, connessa forse coi Mamelucchi (rom. mambrucchi), ci si riferisca» (Formica/Lorenzetti 1999, 489, in nota). ${ }^{19}$ Sembra tuttavia probabile che nel sonetto

18 In assenza di altre attestazioni della voce a Roma - ed escludendo la possibilità di un gergalismo improvvisato - rimane questa una delle conclusioni più economiche. In una prospettiva simile, l'alterazione di pilucche in pilacche potrebbe essere ricondotta a un irrazionale adeguamento alla struttura della fronte (un'influenza dello stesso tipo, ma interna alle quartine, potrebbe giustificare il tricche tricche del verso 5) o più semplicemente a un errore, da parte del copista di B come di altri, nella lettura dell'antigrafo (viene facile pensare all'errata interpretazione di una $<\mathrm{u}>$ tracciata in modo poco chiaro, con la prima asticciola inclinata a tal punto da rasentare la seconda; per esempi di questo tipo si vedano, all'interno dello stesso B, lugubri alla p. 466, luco alla p. 513 o aulivo alla p. 526); simili incidenti, del resto, si spiegherebbero bene guardando proprio alla scarsa diffusione di 'pilucca` nei documenti romaneschi di cui disponiamo (due esempi in totale): se il tipo circolava poco, il suo grado di riconoscibilità doveva essere basso; la probabilità di fraintendimenti, di conseguenza, piuttosto alta.

19 Salvo errore, non s'individuano attestazioni di 'mambrucco' 'Mamelucco' nei più importanti repertori del romanesco, che attestano semmai 'mammalucco' 'idiota, persona sciocca' (cf. Vaccaro 2010, s.v. mammalucco, dove si rinvia a un'occorrenza trilussiana e, poi, a Ravaro e RVRM, s.v.; come nota Ravaro - ma cf. prima Muñoz 1947, 280 - la voce è già in Berneri); con significati diversi, il primo tipo occorre però in Blasi (s.v. mambrùcco 'di persona maldestra, barbara e sudicia'), LDVit (s.v. mambrucco 'di un cavallo scadente; di un individuo stupido'), VCC (s.v. mambrucco 'persona stupida; individuo grezzo ed ignorante’) e VDBl (s.vv. mambrucca 'tipo di pecora; donna di colore; puttana' e mambrucco 'stupido'). Esempi simili si hanno anche nei dialetti umbri documentati da GVS (s.v. mambruccu 'stupido; rozzo'), VTO (s.v. mambrucco 'ingenuo; persona poco socievole; persona mal vestita') e VTT (s.vv. mambrucca 'donna araba' e mambrucco 'ingenuo; persona poco socievole'), ed è interessante notare che il tipo tocca almeno la Calabria a sud (cf. NDDC, s.v. mambrucu 'persona testarda') e, a nord, la Toscana (cf. Fanciulli, s.v. mambrukko 'persona stolta'; Gori/Lucarelli, 
si alluda «[al]la nota canzone» Malbrough s'en va-t-en guerre, brano «in gran voga verso la fine del Settecento, alla Corte di Maria Antonietta» (Trebbi/Ungarelli 1932, 239) e «a Roma tradott[o] con Mambrucche va a la guerra ecc.» (Menarini 1968, 25); della versione capitolina si dà notizia almeno nel Saggio di canti popolari romani di Francesco Sabatini (n. 2; sezione I: Canti politici) e nei Canti popolari romani di Giggi Zanazzo (n. 922; sezione VIII: Canti storici, politici, patriottici). Ecco la canzone così come si legge nei due raccoglitori:

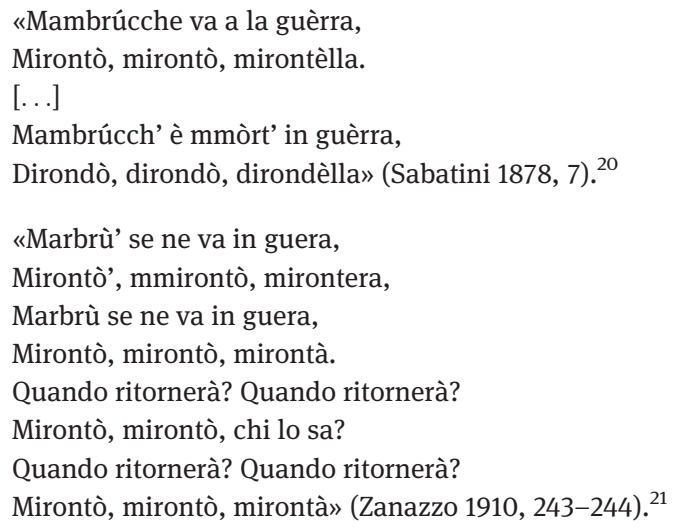

Resta da chiedersi se il sonetto si riferisca alla sola canzone o se celi anche, più sottilmente, un'allusione al successo avuto dalla stessa presso la corte di Maria Antonietta.

\footnotetext{
S.v. mambrucco 'uomo grosso e zotico'; VCort, s.vv. mambrùcca 'donna malvestita, sciatta' e mambrùcco 'uomo rozzo, incivile'; per il fiorentino contemporaneo cf. Poggi Salani et al. 2012, s.v. mambrucco 'scemerello') e l'Emilia (basti il riscontro di Lepri/Vitali, s.v. manbrócc 'zotico, rozzo'). Da notare ancora che mambrucca, -o e varianti dialettali sono registrati, con il significato di 'carrettone, barroccio con ruote alte', in VEI (s.v. mambrucco, che sarebbe la forma attestata a Roma), DEI e GDLI (s.v. mambrucca; anche il DEI dà mambrucco come forma romanesca); la discussione sull'etimo di questa voce è ripercorsa in Zamboni (1986, 106). I dati offerti dai repertori citati sono interessanti e numerosi, ma, come si spera di chiarire a breve, non hanno alcun peso nell'identificazione del nostro aria de Mambrucche.

20 Cf. anche i dati esposti in nota e le osservazioni alle pp. 46-47.

21 Cf. anche i dati esposti a p. 243, in nota; da rilevare che il titolo del testo è, a p. 243, Marbrucche, mentre nell'indice del volume è stampato Mambrucco. Si può osservare qui che lo stesso Zanazzo, in altra sede (Zanazzo 1966, 473), registra Mambrucche tra i soprannomi più comuni a Roma.
} 


\section{Il tricche tracche}

Come per pilacche, anche per tricche tracche si parla, in Lorenzetti (1999b, 157, in nota), di «novità» nel panorama del lessico romanesco; il significato che accompagna la voce è quello di "'oggetto di poco valore (?)'». Nel glossario finale (s.v. tricche tricche, che è la variante del ms. B; cf. il §1 e la nota 6) ${ }^{22}$ si precisa che la parola ha nel nostro testo un «senso oscuro» e che la stessa potrebbe indicare «qcs di poco valore, oppure di tal natura che il rubarla sarebbe un sacrilegio»; poco dopo si ricorda, senza vedere «connessioni plausibili», che «Il tricche tracche è in rom. il 'crotalo', la 'battola' del venerdì santo (Belli, Chiappini), oppure il 'cervello' (Belli)».23

Per 'tricche tracche $\urcorner$ i repertori del romanesco attestano, in effetti, perlopiù i significati di 'battola' (cf. Chiappini, s.v. tricche tracche ${ }^{24}$ VBel, s.v. tricchettracche; Ravaro, s.v. tricchettràcche $;^{25}$ Di Nino 2008, s.v. tricchettracche) e 'cervello' (gergalismo impiegato solo in Belli e debitamente registrato da VBel e Di Nino $2008,{ }^{26}$ nonché da PDG, s.v. trìcche-tràcche); solo Ravaro documenta l'accezione di 'castagnole, serie di piccoli petardi che esplodono in rapida successione, ${ }^{27}$ ma senza esempi. Con forme simili si indica la battola,

22 Non è ben chiaro perché in questo caso non sia stata scelta (e citata nel brano esemplificativo tratto dal componimento) la variante del $\mathrm{ms}$. Y, che è invece impiegata per rappresentare la voce - come ci si aspetta, dato che si tratta della forma stampata a testo - nella Nota linguistica e nel commento al sonetto (l'avvertenza all'inizio del glossario non dà spiegazioni in questo senso). Nel corso del paragrafo ci si riferirà solo a tricche tracche, senza considerare l'alternativa in -icche, che è evidentemente il frutto di una banalizzazione.

23 Un appunto simile, ma senza il riferimento a 'cervello', è nelle note di commento al testo (cf. Formica/Lorenzetti 1999, 489, in nota).

24 Tricche tracche occorre pure nelle Aggiunte e postille di Rolandi (s.v.), che si limitano però a proporre una breve nota integrativa: «in ital. Crepitacolo» (come si vedrà infra, Chiappini aveva glossato con il sinonimo tabelle).

25 Qui il primo esempio è rintracciato ne Il maggio romanesco di Peresio (1688): «Parean soavi el Trunche Trunche più, / E 'l Tricche Tracche con el Zuche zù» (XII, 119, 7-8; cf. Peresio 1688, 434). A dire il vero, Ravaro parla solo di uno 'strumento di legno che, agitato, produce un suono simile a quello delle nacchere', senza allusioni alla settimana santa; per chiarire il significato della glossa è necessario ricorrere agli autori citati dopo Peresio: Belli e Zanazzo (per i brani in questione cf. infra).

26 Da notare che secondo il commento di Vigolo, opportunamente riportato da Di Nino, la parola avrebbe indicato «Propriamente un giuoco d'azzardo, assai comune in quel tempo, il tric-trac, che si faveva [sic] coi dadi e le dame su un tavoliere spartito in due» (cf. Belli 1952, 240; su questo significato si tornerà alla nota 34).

27 Quest'ultimo è il valore con cui la forma ricorre spesso nel Mezzogiorno (si hanno esempi della medesima accezione anche al Nord, ma con minor compattezza; per il Lazio cf. infra a testo): si vedano per es. i dati offerti dai repertori di area campana (limitandosi al napoletano, 
oggi, anche in diversi dialetti laziali a nord e a sud della capitale: così ad Ariccia (DAr, s.v. tric-trac), Bracciano (VDBr, s.v. tricche tracche), Castro dei Volsci (VCVo, s.v. trikkęttrakkę), Manziana (LMan, s.v. tricchetràcche), Norma (PNor, S.v. ttricchettracche) e Viterbo (LDVit, s.v. tricchetracche) ${ }^{28} \mathrm{cf}$. inoltre l'AIS (vol. 4, 789) e l'ALI (vol. 7, 724). ${ }^{29}$ Solo a Civita Castellana compare il significato di 'petardi' (VCC, s.v. tricchetracche). ${ }^{30}$

Come si vede, la documentazione lessicografica non offre novità sostanziali: il quadro delineato da Lorenzetti può essere precisato e ampliato, ma

cf. Andreoli, s.v. tricchitracco; Altamura, s.v. tricchëtràcchë; D’Ambra, s.v. tricchètracco; D'Ascoli, s.v. tricchetracco; solo Volpe, s.v. tricche tracche, attesta, oltre ai significati di 'saltarello', 'razzomatto' e 'tric trac', anche quello di 'raganella'), calabrese (cf. NDDC, s.v. tricchi-tracchi ma anche, s.v., tricchə-troccha 'raganella' - e Martino/Alvaro, s.v. trìcchi-tràcchi), pugliese (cf. Garrisi, s.v. tricchitracchi; Mancarella/Parlangèli/Salamac, s.v. trícchi trácchi; Reho, s.v. tricchittràcche; VDS, s.v. trícchi-trácchi, dove la stessa forma vale anche 'raganella girevole di Pasqua') e siciliana (cf. già Mortillaro, s.v. trìcchi tràcchi, ma soprattutto VS, s.v. ț̣icchitṛacchi ${ }^{1}$, a cui si affianca però țricchițracchi 'crepitacolo; raganella'). Si può aggiungere qui che il VRDR (s.v.) ha solo tricchetetracchete 'camminata' e, nello stesso luogo, l'espressione tricchetetracchete de la settimana santa (senza glosse); è forse inutile precisare che il significato di 'cosa di poco valore' attestato in Di Nino (2008) per il Misogallo romano dipende, ovviamente, dal commento di Lorenzetti.

28 Lo stesso repertorio (s.v.) dà notizia anche di tricchetricche 'incipit di una formula da gioco'. Un utile quadro sulle realizzazioni di 'battola' nella Tuscia viterbese si legge in Cimarra/Petroselli (2002, 6509), con descrizione dello strumento e del suo impiego durante la settimana santa.

29 Il primo attesta forme affini a tricche tracche nei punti 58 (Poschiavo), 160 (Pontechianale), 261 (Milano), 618 (Castelli), 637 (Capestrano), 640 (Cerveteri), 648 (Fara San Martino), 652 (Roma), 658 (Palmoli), 736 (Matera), 738 (Avetrana), 742 (Acquafredda, presso Maratea), 751 (Acquaformosa) e 896 (Giarratana); il secondo - ma qui, vista la mole della documentazione, ci si limita ai luoghi più significativi - registra lo stesso tipo nei punti 617 (Montalto di Castro), 620 (Viterbo), 641 (Cerveteri), 642 (Riano), 662 (Roma), 666 (Ferentino), 680 (Ardea), 684 (Casalattico), 689 (Nettuno), 690 (Latina), 692 (Pastena) e 695 (San Felice Circeo).

30 Quanto ai repertori dell'italiano, il valore di 'raganella, crepitacolo della settimana santa' è attestato per il romanesco dal GDLI (s.v. tricchetracche/tricchitracchi), che cita però, al proposito, un esempio dalle Rime piacevoli del fiorentino Fagiuoli (il brano è accolto già nel TB, s.v. tric trac/tricche tracche; al Fagiuoli è ricondotta la prima occorrenza di tricche tracche nel DEI, s.v.) riferito - come si ricava dagli stessi TB e DEI - a un rumore di passi; l'inesattezza di collocazione dipende forse da un cattivo scioglimento dell'abbreviazione che accompagna l'esempio nel vocabolario del Tommaseo (Rim., letto Rom. 'romanesco'). Per i significati assunti da tric trac, tricche tracche etc. in italiano e in alcuni dialetti cf. i già citati $\mathrm{TB}$ (s.v. tric trac/tricche tracche), DEI (s.vv. tricche tracche, tricchi-tracchi, tric trac ${ }^{1}$ e tric trac ${ }^{2}$ ) e GDLI (s.vv. tricchetracche/tricchitracchi, tricchitracchi e trictrac), a cui aggiungere DELI (s.v. tric-trac; qui il significato di 'raganella della settimana santa' emerge solo nella sezione etimologica) ed EVLI (s.v. tric-trac/trìcche-tràcche; qui la parola vale solo 'gioco della tavola reale'). 
senza acquisizioni di rilievo; né s'individuano esempi di 'tricche tracche 'oggetto di poco valore'. Sul versante letterario le cose non vanno meglio: oltre ai significati di cui dànno conto i dizionari citati supra ('battola'; 'cervello'), s'incontrano due altri valori che, però, hanno ben poca utilità ai nostri fini: in un sonetto di Paolo Mereghi tricche e tracche è riferito al pulsante del telegrafo: «Si voi telegrefà, Rosa, hai da sbatte / quer tricche e tracche, fatto a ponticello» (Er telegrifo senza li fili, 5-6; cf. Mereghi 1951, 45); indicano più banalmente un suono - di ossa nel primo caso, di chiavi nel secondo - le occorrenze di tricche tracche ne La morte imbriaca di Egisto Olivieri (1; cf. Possenti 1966, 460) e in Tramonto ne' la basilica di Giulio Cesare Santini (58; cf. Santini 1962, 265). ${ }^{31}$

In verità, la soluzione del problema potrebbe trovarsi non in una nuova attestazione o in una glossa ad hoc (quella, cioè, elaborata dubitativamente dal commentatore), ma in ciò che già sappiamo: sembra infatti ragionevole ritenere che il significato di 'crotalo, battola' non vada escluso dal novero delle possibilità interpretative e che lo stesso, al contrario, possa adattarsi bene all'occorrenza misogallica. Del resto, un'interpretazione simile avrebbe il pregio di combinare e valorizzare proprio le due intuizioni esposte dal curatore del glossario: come si vedrà meglio più avanti, la battola era un oggetto di scarso valore dal punto di vista materiale, ma di una certa importanza sul piano liturgico; un oggetto del cui furto ci si poteva legittimamente stupire o lamentare e che, per questo, nel contesto dei versi 5-6 non si troverebbe fuori posto.

Fortunatamente, gli autori romani offrono qualche brano interessante in questo senso. Tralasciando il tricche tracche di Peresio, il cui contesto è stato già citato alla nota 25, vale la pena di menzionare anzitutto l'occorrenza belliana del sonetto 878 (Er Venardì Ssanto), in cui il poeta ricorda l'usanza di far chiasso, per commemorare la passione di Cristo, con «mazzole / e [. . . tricchettracche e rraganelle / che sse fanno, pe ddillo in du' parole, / de leggno, ferro, canna, crino e ppelle» (5-8). Una descrizione più accurata dello strumento si ha, verso la fine dell'Ottocento, ne Er tricche tracche di Filippo Tolli (in Possenti 1966, 208):

«Er tricche-tracche, Cencio, è quer congegno

bislongo, inerto, arto tre parmi e piano,

fatto cor una tavola de legno

e un bucio in cima p'infilà' 'na mano.

È lavorato senz'arcun disegno;

meno che sopra l'uno e l'antro vano

31 Privo di rilievo il soprannome Tricchetracche ne Er corpo gobbo (V, 9; XVI, 2) di Fabio Della Seta (2001, 178 e 184). 


\begin{abstract}
c'è un fero che, si trittichi l'ordegno, lo senti cento mija da lontano. Questo è l'unico segno ch'arimane ar chirichetto che lo sbatte forte quanno che so' legate le campane.

Esce de chiesa, fa un giretto intorno, e, tiranno li serci pe' le porte, avvisa ch'è sônato mezzoggiorno».
\end{abstract}

L'impiego dell'idiofono è argomento anche per Zanazzo (1908, 219), che racconta come «dar giovedì ar sabbito santo, er mezzogiorno, l'ore de la messa e dde ll'antre funzione de le cchiese, li chirichetti le sonàveno defôra de la cchiesa, co' li tricche-tràcche»; stando allo stesso autore (La Pasqua a Roma, XXIX, 1-4), il suono dei «tricche tracche» si aggiungeva poi a quello prodotto da «mazzolate, / [. . . bussolotti, / zaganelle, schioppettate, / mortaletti, razzi, botti» nel festoso e assordante concerto che seguiva lo scioglimento delle campane (cf. Zanazzo 1968, 534). ${ }^{32}$

Gli esempi non sono molti, ma se ne può trarre un buon profitto: dai brani citati, infatti, s'intuisce bene quale fosse l'importanza del tricche tracche, strumento modesto (se ne rileggano le descrizioni) a cui, però, si trasferivano le funzioni delle campane in uno dei periodi più seri e rilevanti nella liturgia della comunità cristiana (da notare anzi ciò che scrive Tolli: «Questo è l'unico segno ch'arimane / ar chirichetto»; corsivo aggiunto); ed è per questo notevole che il sonetto di Belli parli, a proposito del baccano originato nel venerdì santo da mazzole, battole e raganelle, di un vero e proprio voto: «Er chiasso che cce fâmo è stato un voto / per immità cco li su' soni veri / cuello der temporale e 'r terramoto [scil. i fenomeni atmosferici verificatisi in coincidenza con la morte di Cristo]» (9-11). Una testimonianza ancor più significativa viene dal Vocabolario romanesco di Filippo Chiappini (qui già citato: cf. supra); alla glossa della voce tricche tracche, infatti, il lessicografo romano fa seguire la descrizione di un episodio piuttosto interessante:

32 Forse alla battola si riferisce anche un'altra attestazione zanazziana - parte di un dialogo imprecatorio - di interpretazione più incerta: «- Te piji un accidente sotto a le zinne, accusì te va indietro e' latte. - Ma ne li cannèlli de li stinchi accusì caschi a faccia avanti! - Ma in de li fianchi, accusì fai da tricche-tracche» (Zanazzo 1966, 434). Il paragone potrebbe essere proprio tra lo strumento - i cui componenti suonano se agitati, in direzioni opposte, con scatti frequenti della mano - e l'andamento della persona colpita da un malanno ai fianchi, nonché, forse, tra il suono secco e ripetitivo dell'idiofono e quello che potrebbero produrre le articolazioni o le ossa della stessa (come si è visto supra, tricche tracche è impiegato da Egisto Olivieri in riferimento a un rumore di ossa). 
«Tricche tracche, Tabelle [. . .]. Molti anni fa in occasione di un matrimonio tra due vecchi ridicoli, una comitiva di mattacchioni andò a far loro la serenata. Tra i varii strumenti ch'essi avevano portato per fare il chiasso c'era anche il tricche tracche. Il direttore d'orchestra, postosi in mezzo ai sonatori, cantava una filastrocca lì per lì improvvisata, e alla fine d'ogni verso chiamava lo strumento che doveva accompagnarlo [...]. Così [...] venne la volta del tricche tracche. Il direttore intonò:

Seguitiamoli alle tacche

e chiamò il tricche tracche.

- Oh questo poi no - gridò un collarone che per caso s'incontrò a passare. - Oh questo poi no! Il tricche tracche è strumento sacro!» (Chiappini, s.v. tricche tracche)

Un collarone, cioè - come si evince dallo stesso Chiappini (s.v. collaróni) - un fratello «dell'oratorio del p. Caravita», si oppone alla profanazione di uno strumento all'apparenza insignificante, ma evidentemente pregno di connotazioni simboliche per i cittadini più devoti. L'importanza documentaria della storiella non va sovrastimata (com'è evidente, si tratta di un aneddoto arguto); è però chiaro, ancora una volta, quanto stretto fosse il rapporto che legava il nostro piccolo strumento all'àmbito sacro: si trattava di fatto di un vero e proprio elemento liturgico.

Questa breve rassegna sembra offrire spunti sufficienti per ribadire quanto si scriveva più sopra: la possibilità che nel nostro testo si alluda alla battola non può essere scartata. ${ }^{33}$ Il furto del tricche tracche si presenta, difatti, come l'azione inutile e sfrontata di un ladro che non si dà alcun limite e che, alla propria avidità, non sa opporre nemmeno il rispetto dei riti religiosi: è evidentemente assai poco giustificata l'intenzione di chi spera di poter rubare, insieme a molte altre cose, persino il grossolano strumento che richiama i fedeli alle cerimonie connesse con la passione di Cristo (e che, come attesta Belli, nel pomeriggio del venerdì santo annuncia la morte di Cristo stesso). L'assurdità di un

33 Tra l'attestazione misogallica e quelle di Belli, Tolli, Zanazzo e Chiappini vi è indubbiamente una certa distanza temporale, ma l'uso di strumenti di legno in sostituzione delle campane, come ricorda anche Baroncini (2005, 86, in nota), è descritto già nella berneriana Poesis jocosa («una sorta di raccolta di tradizioni popolari romanesche ante litteram» secondo Costa 2017, 4; sull'opera cf. da ultimo Cristelli 2018), precisamente nel brano intitolato Describuntur Pueri, qui in ultimis Majoris Hebdomadæ diebus ligneis malleis pulsant januas domorum; \& facto Vespere, pulsant Gradus Sacrorum Templorum (cf. Berneri 1715, 32-35). Molto interessante l'accostamento tra il sonetto di Tolli e quel che si legge, per l'appunto, in Berneri; cf. in particolare i versi 7-16: «Non sonus auditur, quo nos ad Templa vocamur, / Et prope, qui tempus Vesperis, esse monet. / Præ mœrore silent; supplent quæ lignea, causant / Instrumenta sonum, his Vesperis hora datur. / Est tabula ad geminos palmos producta; sed unum / Per palmum lata est; quæ leve pondus habet. / Hanc puer impugnat dextra, extat quippe foramen, / Aptatur facilis cui bene clausa manus. / Inde citus properans agitat versatile lignum; / Ferrea sic edunt pondera mota sonum» (Berneri 1715, 32-33). 
gesto simile si comprende bene soprattutto se si considera che l'impiego della battola rientrava nel complesso sistema di rituali simbolici che s'incrociavano nella celebrazione del triduo pasquale (tale impiego trovava in passato, tra l'altro, precise interpretazioni da parte dei liturgisti: cf. Baroncini 2005, 86).

Non pare azzardato, insomma, valorizzare il significato di 'battola', che consente oltretutto la soluzione più economica in termini lessicografici; glossando in tal modo, infatti, 1) il senso non manca: i Francesi sarebbero pronti a rubare di tutto, persino l'innocuo quanto fondamentale strumento con cui nella settimana santa, in assenza delle campane, i chierichetti annunciano le funzioni (tenendo conto dello spirito reazionario che anima il Misogallo romano operazione di chiaro «humus curiale»; cf. Lorenzetti 1999b, 108 - l'affermazione non dovrebbe stupire); 2) si può mantenere il significato con cui la voce ricorre, di frequente, nei repertori e negli autori di Roma (e, come si è visto, nelle raccolte di area laziale), senza formulare altre accezioni; 3) come si anticipava, si risponde proprio alla proposta interpretativa - perfettamente condivisibile - di Lorenzetti, dato che la glossa 'strumento umile ma simbolicamente importante, insostituibile (perché, in effetti, già sostitutivo delle campane)' concorda bene con ciò che si nota nel glossario dell'edizione: «Dal contesto si indovina essere qcs di poco valore, oppure di tal natura che il rubarla sarebbe un sacrilegio».

A ben vedere, la connessione tra il senso ordinario della voce in romanesco e quello del nostro sonetto non solo è plausibile, ma, di più, si concilia bene con il tema della miscredenza francese, così vivo nei brani del Misogallo romano (cf. Formica 1999, 70-74 e 82). ${ }^{34}$

34 Ciò non significa che la soluzione sia necessariamente questa; la proposta vuole soprattutto invitare alla cautela, mostrando che il significato più noto della forma può dare senso e che quindi, forse, è possibile precisare l'accezione iperonimica di 'oggetto di poco valore' fornita dal commento. Quanto ad altre, possibili interpretazioni, sarà bene notare che il tricche tracche del testo 407 potrebbe indicare anche, semplicemente, la tavola su cui si giocava il tric trac (o tavola reale, per cui cf. già le note 26 e 30; la voce non è attestata in romanesco, ma circolava in francese e in italiano): in tal caso, e se fà policche avesse davvero il significato di 'far piazza pulita' (cf. il paragrafo seguente), i versi 5-7 acquisterebbero un discreto senso: '(i Francesi) vogliono rubare persino la tavola del tric-trac, e vogliono fare piazza pulita (giocando) di quanto c'è di buono, riempiendosi le sacche'. Un risultato simile si otterrebbe, e con maggior sicurezza, se si riuscisse a legare policche al fr. poule, visto che, come si vedrà alla nota seguente, faire une poule era espressione impiegata anche nella tavola reale per indicare l'azione di chi giocava una partita dopo aver fissato una posta; ma il rapporto tra fà policche e faire une poule - e lo si ribadirà - è più che incerto. Ad ogni modo, è evidente che la glossa 'tavola reale' non potrà essere discussa finché non sarà stato verificato, con certezza, il senso di fà policche. 


\section{Una nota (provvisoria) su fà policche}

Veniamo a fà policche. Il commento al testo si limita a notare che la locuzione è «senza riscontri» e «vale forse 'far piazza pulita'» (Formica/Lorenzetti 1999, 489, in nota); nel glossario (s.v. policche) si aggiunge che policche «Non [è] in Belli». Posto che non è stato possibile rintracciare dati pienamente soddisfacenti, ${ }^{35}$ ci si limiterà a segnalare un'analogia - curiosa ma, per l'appunto, non risolutiva - con due voci attestate nei territori di Todi e Subiaco.

Nel VTT, dunque, si registra (s.v.) una parola che ricorda da vicino l'occorrenza del sonetto: si allude a pulicche, la cui affinità con policche (e pelicche) spicca tanto sul piano della forma quanto - se si accoglie la glossa di Lorenzetti - su quello del significato, visto che della voce si dà conto nella loc. avv. $a$

35 Considerati il tipo di testo e la terminazione in -icche, si potrebbe intravedere nell'occorrenza misogallica un francesismo; finora, però, non sono emerse forme galloromanze capaci di spiegare policche (o pelicche, che è la variante del ms. Y: cf. il §1). È suggestiva, ma molto artificiosa e in ogni caso difficilmente verificabile - l'ipotesi di un conio iperfrancesizzante, vale a dire di un utilizzo abusivo delle strutture del francese in funzione parodica: nel concreto, l'autore del sonetto potrebbe essere partito da una voce esistente e aver quindi scimmiottato, su pressione della rima, una caratteristica terminazione d'Oltralpe; di procedimenti simili si hanno in effetti altri esempi, e qui si potrebbe citare almeno il Raguet di Scipione Maffei (1747), commedia interamente dedicata alla ridicola mimesi del francese nel parlato quotidiano e caratterizzata, passim, dalla creazione di forme aberranti mediante moduli tipici della lingua oggetto di accusa (esempi: «Non si potrebbe incivilir, dicendo: / Santò, Moscatellác, Monpulcianò?» nell'atto III, scena II; «Ecco che mi ricordo ancor di quando, / perché non seppi dir doré e giallò, / voi mi deste de’ piedi nel culò» nell'atto IV, scena VI; «vivrò [. . . ] / con la mia sospirata Ersilión [per Ersilia]» nell'atto V, scena VI; cf. Avena 1928, rispettivamente alle pp. 192, 211 e 220). Tra le basi accreditabili, in un caso simile, si dovrebbe citare almeno il fr. poule, voce che compare per la prima volta nel 1665 con il significato di 'quantité d'argent ou de jeton qui résulte de la mise de chacun des joueurs et qui appartient à celui qui gagne le coup' (cf. TLF, s.v. poule ${ }^{2}$; l'occorrenza è ne La maison des jeux académiques); nella fattispecie, le locuzioni faire, jouer une poule sono attestate già nella terza edizione (1740) del DAF (s.v. poule), dove se ne registra l'impiego nel gioco del «Trictrac, \& à quelques autres jeux» con il significato di «Faire une partie où tous les joueurs mettent une certaine somme chaque fois qu'ils entrent au jeu, \& qui demeure en total à celui qui a gagné tous les autres de suite». Se policche fosse l'adattamento di un fittizio [pu'lik], in breve, si potrebbe interpretare con un sensato 'fare posta, giocarsi, puntare al gioco' (meno ovvia la presenza dell'o protonica, che dovrebbe trovare una giustificazione ragionevole; ancor più difficile, evidentemente, spiegare pelicche); a favore di una simile ricostruzione parlerebbero almeno due elementi: 1) l'affinità fraseologica che lega fà policche a faire une poule e 2) la presenza nel sonetto della forma tricche tracche (tricche tricche in B; cf. il §1), dato che, come si è visto, la loc. faire une poule è associata nel DAF anche al gioco del tric-trac (cf. già la nota precedente). Questi elementi, però, non controbilanciano l'onere complessivo dell'ipotesi, che resta assai poco verosimile. 
pulicche 'al verde, senza soldi' (il dato è anche in UDia, s.v. pulà; la fonte sarà proprio il repertorio di Ugoccioni e Rinaldi). Un esempio simile s'incontra nell'alta Valle dell'Aniene, dove a Subiaco è documentata l'espressione te manno a puliccu 'ti mando fallito (in forma lieve)' (cf. PVSub, s.v. puliccu; nello stesso repertorio, s.v., anche puliccà 'sottrarre a poco a poco, vincere denaro all'avversario', che è ricondotto a piluccare - ma questo verbo manca al vocabolario - e di cui si registra il participio puliccatu).

A loro volta, le espressioni del todino e del sublacense sembrano confrontabili con una serie di locuzioni presenti in alcuni dialetti dell'Italia centrale e indicanti per l'appunto la perdita o l'acquisto di una significativa quantità di denaro (in massima parte in relazione al gioco): limitandosi a un semplice campione, si potrebbe rinviare a VDBl (s.v. pula; a la pula 'al verde' e 'del tutto'), VDMa (s.v. pula'; èss a la, al pula e armandè ta ùn a la pula 'essere al verde'), VDTi (s.v. pulu/pullu; mannà a pulu 'far rimanere al verde con il gioco', ì a pulu 'perdere la partita, rimaner pulito'; e cf. DTiv, 163b) e, tornando al todino, VTT (s.v. pula'; mannà a ppule 'ripulire le tasche, ridurre al verde' a Todi, ${ }^{36}$ rimané a le ppule 'rimanere senza soldi; rimanere senza briscola' a Fratta Todina; si ha anche pula 'denaro' con la frase nun c'ha più la pula); locuzioni congeneri sono allegate dal LEI (vol. 7, 1491 e 1494), che pensa a costrutti con 'pula' 'involucro che si stacca dai semi di cereale con la trebbiatura; materiale di scarto dei cereali' ${ }^{37}$

36 L'espressione è marcata come arcaica e scherzosa.

37 I repertori dialettali documentano talora espressioni simili a quelle riportate a testo, ma per le quali è più difficile stabilire se si muova da 'pula ${ }^{\top} \mathrm{o}$ da un altro tipo. Qui non è possibile affrontare nel dettaglio il problema, che pare piuttosto complesso e che richiederebbe, perciò, ben altre energie; ci si limiterà a notare che, almeno per alcune locuzioni, non sembrano da escludere interventi manipolativi a partire da 'pula ${ }^{\top}$ o contatti tra 'pula ${ }^{\top}$ e tipi a quest'ultimo affini: cf. ad es. il VVPi (s.v. pulì; èsse a pulì e pulà 'aver perduto tutto') e il VMar (s.v. pulìmme; mandà a le pulìmme 'vincere tutto'), ma anche il VDBl (s.v. pelù, $a$; mannà a pelù 'vincere al gioco') e il VDFR (s.v. pelù; mann-à ppelù 'vincere continuamente denaro al gioco'; l'autore rinvia ai sinonimi spelusà e spolinà). Almeno sulla carta, per le forme viterbesi annà a le peluce, a le pilucis 'perdere tutto al gioco; andare in miseria' (cf. DIVVI, s.vv. pelùce, annà à lé $\mathrm{e}$ pilùcis, annà à lé) valgono due spiegazioni: da un lato, si può trattare di espressioni indipendenti da 'pula', costruite con derivati del v. spelucià, spi- 'vincere al gioco tutto il denaro di uno o più avversari' (magari in una forma, non documentata, priva dell's-; cf. comunque DIVVI, s.v. spilucià-spelucià) secondo uno schema che per la lingua del gioco è attestato, tra l'altro, anche in romanesco (si pensi ad annà a lo spelliccio 'perdere ogni cosa', dove spelliccio è un deverbale da spelliccià 'spogliare un giocatore, vincergli tutto'; cf. Fresu 2010, 277); dall'altro, non si può escludere che le stesse dipendano da un contatto tra derivati di spelucià, spi- ed espressioni con 'pula' come quelle raccolte più sopra (cf. del resto DIVVI, s.v. pùle, annà à lé; com'è chiaro, un'interferenza simile spiegherebbe l'assenza dell's-). Si tenga comunque presente, vista la serie di esempi citati in questa nota, che lo svisamento di una parola 
Ora, è necessario chiedersi se, soprattutto da un punto di vista formale, sia effettivamente possibile collegare fà policche, pe- agli esempi dialettali citati poc'anzi, confermando così la già assai persuasiva glossa di Lorenzetti ('ripulire, fare piazza pulita'). In questo senso, è possibile proporre qualche breve osservazione.

L'ostacolo più significativo al collegamento proposto è, come risulta evidente, la diversa costruzione delle locuzioni prese in esame: da un lato fare (policche, pe- di qualcosa); dall'altro andare $a$, essere $a$, mandare a e rimanere $a$. L'incongruenza è notevole e, di fatto, pare sufficiente a mettere in guardia circa la possibile origine comune delle forme: per giustificarla si potrebbe tuttalpiù supporre un incontro - ipotesi motivabile, forse, con ragioni metrico-rimiche - con altre locuzioni simili. Di tali locuzioni, tuttavia, se si eccettua l'it. fare pulizia 'consumare con voracità, dilapidare' (GDLI, s.v. pulizia'), non è possibile reperire esempi degni di rilievo. Da escludere, per es., fà piazza pulita, che pure compare già in Micheli secondo Ravaro, s.v. piàzza. E poco rilevante sembra anche il confronto con espressioni sì costruite con fare, ma impieganti un infinito: si pensi ad es. a fà poli/pulì ppròprio 'prendere tutto', registrata nel VDBl, s.v. polì; fann'a polì/pulì 'fanno tabula rasa', nel LDVit, s.v. polì; f-à ppolì, - a testo ppoli, per un refuso 'cogliere tutti i frutti di un albero', nel VDFR, s.v. poli. Diversamente, si dovrebbe ammettere uno sviluppo per cui il solo pulicche (o varianti; sul vocalismo protonico delle forme citate si veda infra) avrebbe assunto autonomia, fuori di locuzione, con il significato di 'rovina, tabula rasa'.

Evidentemente più semplice sarebbe spiegare il vocalismo protonico delle attestazioni misogalliche ( $o$ in $\mathrm{B}, e$ in $\mathrm{Y}$ contro $u$ del todino e del sublacense, supponendo che siano questi ultimi - viste le sopraccitate locuzioni con pula, -e, - $u$ - a presentare la condizione originaria). Nel caso di policche, la presenza della media, già di per sé banale, potrebbe essere agevolmente ricondotta ad oscillazioni fisiologiche in un regime in cui il passaggio di /o/ protonica a $/ \mathrm{u} /$ era già (ben) rappresentato (cf. Lorenzetti 1999b, 145) o, ancora, ad un abbassamento di tipo "laziale" (cf. ad esempio RVRM, VI). Quanto a pelicche, non sarebbe obbligatorio supporre un'alterazione di natura testuale: la forma, infatti, potrebbe trovare una giustificazione considerando le espressioni registrate alla nota 37, ossia presupponendo l'interazione di tipi lessicali diversi (nella fattispecie, un incontro con 'pelare 7 ). Per lo stesso policche, del resto, sarebbe possibile ipotizzare un contatto con forme di 'pulire ${ }^{\urcorner}$aventi la media in protonia; nella

sulla base del confronto con voci affini (per «convergenza semantico-formale» o soltanto «formale») è un processo frequente nel rimaneggiamento linguistico d'àmbito gergale (cf. Ageno 1957, 412-419; sui possibili rapporti tra le locuzioni in questione e l'ambiente gergale, che motivano questo appunto, cf. infra). 
documentazione di area romana si ha solo polita, -o 'di alta qualità' - due esempi nella Cronica, rispettivamente ai capp. XVIII, 680 e XXVII, 378b -, ma l'o è nota ancor oggi a diversi dialetti laziali (cf. a titolo d'esempio LDVit, s.vv. poli, polita, politezza, polito e politrice; VCor, s.vv. poli', polito, -a e politura; VDTi, s.vv. poli, polisse e politu; cf. anche l'AIS, vol. 8, 1551).

Da aggiungere infine, più in generale, una considerazione relativa a -icche, $-u$. Per queste uscite è certo possibile notare con Rohlfs (1966-1969, §1048), rispetto al quale pochissimo aggiunge Tekavčić (1972, §1885), che i suffissati in -icco sono piuttosto rari nella lingua nazionale e nei dialetti; ciò che sembra utile evidenziare qui, però, è che per spiegare la genesi di voci come pulicche, $-u$ (e quindi, forse, policche, pe-) potrebbe bastare l'ipotesi - ma non andranno escluse altre ricostruzioni $-{ }^{38}$ di un trattamento deformante di natura gergale: a una dinamica simile farebbero pensare ancora una volta le locuzioni citate alla nota 37; ed è d'altronde il contesto d'uso di pulicche, $-u$, vale a dire quello del gioco, che autorizza a postulare degli interventi analoghi a quelli descritti per esempio in Ageno (1957, 406408) o in Sanga (1993, 162-163).

In conclusione, al possibile legame tra policche, pe- e pulicche, -u osta principalmente l'assenza, tra le locuzioni elencate più sopra, di costrutti con fare; la somiglianza tra le forme potrebbe essere banalmente poligenetica. L'ipotesi di una caricatura del francese (cf. la nota 35) è priva di sostanza documentaria; liquidare quest'eventualità sarebbe d'altra parte incauto, visto che non si hanno alternative dirimenti e che una spiegazione simile, da un punto di vista extralinguistico, si attaglia al tenore del sonetto e al suo orientamento francofobo: nuove riflessioni potranno forse identificare rapporti utili con forme galloromanze sfuggite a chi scrive. In attesa di intuizioni o di confronti risolutivi, il dibattito sulla natura di policche (pelicche?) resta aperto.

38 In linea di principio, ad es., si potrebbe anche partire da un derivato zero di un verbo come puliccà, forma sublacense registrata più sopra (del resto, si è già incontrato il deverbale romanesco spelliccio: cf. la nota 37); per il momento questa soluzione si rivela però difettosa, perché puliccà - salvo sviste - è registrato nel solo PVSub e dunque puliccu può essere tanto la forma di arrivo quanto quella di partenza. Va inoltre sottolineato che la sequenza [ikk], se da un lato suggerisce la possibilità di un modellamento di tipo puramente gergale (cf. infra nel capoverso) o di un intervento indotto in via estemporanea dalla necessità di rispettare una rima difficile (spiegazione valida, naturalmente, per i soli policche, pe-, che andrebbero allora separati dalle forme centro-italiane succitate), dall'altro non può non ricordare verbi quali rom. spollicchià, spulicchià 'rovinare al gioco, ripulire' (cf. Ravaro, s.vv.): forme simili potrebbero essere coinvolte, in qualche misura, nella nascita delle voci qui discusse (o di alcune di esse, qualora non si volesse ammettere una relazione tra pulicche, $-u$ e le occorrenze misogalliche). 


\section{Bibliografia}

Ageno, Franca, Per una semantica del gergo, Studi di filologia italiana 15 (1957), 401-437. AIS = Jaberg, Karl/Jud, Jakob, Sprach- und Sachatlas Italiens und der Südschweiz, 8 voll., Zofingen, Ringier, 1928-1940.

$\mathrm{ALI}=$ Atlante linguistico italiano, Roma, Istituto Poligrafico e Zecca dello Stato/Libreria dello Stato, 1995ss.

Altamura = Altamura, Antonio, Dizionario dialettale napoletano, Napoli, Fiorentino, ${ }^{2} 1968$. Andreoli = Andreoli, Raffaele, Vocabolario napoletano-italiano, Torino, Paravia, 1887.

Avena, Antonio (ed.), Scipione Maffei, Opere drammatiche e poesie varie, Bari, Laterza, 1928. Baroncini, Rodolfo, L'ufficio delle Tenebre. Pratiche sonore della settimana santa nell'Italia settentrionale tra Cinque e Seicento, Recercare 17 (2005), 71-134.

Belli, Giuseppe Gioachino, I sonetti, ed. Vigolo, Giorgio, 3 voll., Milano, Mondadori, 1952.

Belli, Giuseppe Gioachino, Tutti i sonetti romaneschi, ed. Teodonio, Marcello, 2 voll., Roma, Newton Compton, 1998.

Belloni, Pietro/Nilsson-Ehle, Hans, Voci romanesche. Aggiunte e commenti al Vocabolario romanesco Chiappini-Rolandi, Lund, Gleerup, 1957.

Beltrami, Pietro G., La metrica italiana, Bologna, il Mulino, ${ }^{5} 2011$.

Berneri, Giuseppe, Poesis jocosa, seu morum ac ludicrorum quorundam, quæ olim Romæ; modò vero tum apud Romanos, tum apud nostrates vigent poeticæ descriptiones, Patavii, apud Josephum Corona, 1715.

Blasi = Blasi, Bruno, Il dialetto cornetano, Bollettino della Società Tarquiniense d'Arte e Storia 12 (1983), 1-67.

Bompadre, Giacomo Palmiro, Ar mercato. Sonetti romaneschi, Roma, Ferri, 1978.

Chiappini $=$ Chiappini, Filippo, Vocabolario romanesco, ed. Migliorini, Bruno, con aggiunte e postille di Ulderico Rolandi, Roma, Chiappini Editore, ${ }^{3} 1967$.

Cimarra, Luigi/Petroselli, Francesco, Proverbi e detti proverbiali della Tuscia viterbese. Commento, lemmario italiano, indice semasiologico, riscontri con raccolte edite, indice numerico, Viterbo, Cultura subalterna, 2002.

Costa, Claudio (ed.), Benedetto Micheli, Povesie in lengua romanesca, Roma, Edizioni dell'Oleandro, 1999.

Costa, Claudio, La satira in Roma in età moderna, 2017, consultabile all'indirizzo www.galan tara.it/Ricerche/argomenti/SatiraRoma_CCosta.pdf [ultimo accesso: 21.3.2019].

Cristelli, Stefano, /l Berneri latino e le «loquutiones adolescentium Romanæ Plebis», Lingua e Stile 53 (2018), 155-166.

D’Achille, Paolo, Recensione a Formica, Marina/Lorenzetti, Luca (edd.), Il Misogallo romano, prefazione di Tullio De Mauro, Roma, Bulzoni, 1999, Rivista italiana di dialettologia 33 (2009), 362-364.

$\mathrm{DAF}=$ Dictionnaire de l'Académie françoise, 2 voll., Paris, Coignard, ${ }^{3} 1740$.

D'Ambra = D'Ambra, Raffaele, Vocabolario napolitano-toscano domestico di arti e mestieri, Napoli, presso l'autore, 1873 (ristampa anastatica Sala Bolognese, Forni, 1996).

$\mathrm{DAr}=$ Leoni, Mario, Il dialetto di Ariccia, Ariccia, Comune di Ariccia, 1999. 
D’Ascoli = D'Ascoli, Francesco, Nuovo vocabolario dialettale napoletano. Repertorio completo delle voci, approfondimenti etimologici, fonti letterarie, locuzioni tipiche, presentazione di Nicola De Blasi, Napoli, Gallina, 1993.

$\mathrm{DEI}=$ Battisti, Carlo/Alessio, Giovanni, Dizionario etimologico italiano, 5 voll., Firenze, Barbèra, 1950-1957.

DELI = Cortelazzo, Manlio/Zolli, Paolo, Dizionario etimologico della lingua italiana, 5 voll., Bologna, Zanichelli, 1979-1988 (nuova edizione in unico vol. con il titolo /l nuovo etimologico, edd. Cortelazzo, Manlio/Cortelazzo, Michele A., 1999, da cui si cita).

Della Seta, Fabio, Roma in valigia. Mille e anche più sonetti in Urbe et in Orbe, Roma, Stango, 2001.

DIDE = Cortelazzo, Manlio/Marcato, Carla, I dialetti italiani. Dizionario etimologico, Torino, Utet, 1998.

Di Monaco, Florindo, Un mondo di acconciature. Itinerario alla scoperta di centomila idee e misteri che vengono quotidianamente al pettine, Napoli, Edizioni Scientifiche Italiane, 1999.

Di Nino, Nicola, Glossario dei sonetti di G.G. Belli e della letteratura romanesca, presentazione di Luca Serianni, Padova, Il Poligrafo, 2008.

DIVVI = Galeotti, Vittorio/Nappo, Fiorenzo, Dizionario italiano-viterbese viterbese-italiano, Viterbo, Sette città, 2005.

DTiv = Sciarretta, Franco, /l dialetto di Tivoli. Nascita e sviluppo dall'età classica ad oggi, Tivoli, 1999.

EVLI = Nocentini, Alberto, l'Etimologico. Vocabolario della lingua italiana, con la collaborazione di Alessandro Parenti, Firenze, Le Monnier, 2010.

Fanciulli = Fanciulli, Pietro, Vocabolario di Monte Argentario e Isola del Giglio, Pisa, Giardini, 1987.

Formica, Marina, Introduzione, in: Formica, Marina/Lorenzetti, Luca (edd.), Il Misogallo romano, prefazione di Tullio De Mauro, Roma, Bulzoni, 1999, 9-106.

Formica, Marina/Lorenzetti, Luca (edd.), Il Misogallo romano, prefazione di Tullio De Mauro, Roma, Bulzoni, 1999.

Fresu, Rita, Stratificazione e tipologia del lessico italoromanzo dei giochi di carte. Primi sondaggi, Annali della Facoltà di Lettere e Filosofia dell'Università di Cagliari 65 (2010 [2011]), 263-282.

Garrisi = Garrisi, Antonio, Dizionario leccese-italiano, 2 voll., Cavallino di Lecce, Capone, 1990. GDLI = Battaglia, Salvatore/Bàrberi Squarotti, Giorgio (edd.), Grande dizionario della lingua italiana, 21 voll., Torino, Utet, 1961-2002 (con 2 suppl., ed. Sanguineti, Edoardo, 2004 e 2009).

Giorgetti, Cristina, Manuale di storia del costume e della moda, Firenze, Cantini, 1993.

Gori/Lucarelli = Gori, Lidia/Lucarelli, Stefania, Vocabolario pistoiese, ed. Giacomelli, Gabriella, Pistoia, Società Pistoiese di Storia Patria, 1984.

GVS = Cuzzini Neri, Giampiero/Gentili, Lamberto, Grande vocabolario del dialetto spoletino (1972-2008), presentazione di Enzo Mattesini, Spoleto, Opera del Vocabolario dialettale umbro/Nuova Eliografica Editore, ${ }^{2} 2009$.

Incarbone Giornetti, Rossella (ed.), Benedetto Micheli, La Libbertà Romana acquistata e defesa. Povema eroicomico in dialetto romanesco del sec. XVIII, Roma, A. S. Edizioni, 1991.

LDVit = Petroselli, Francesco, Il lessico dialettale viterbese nelle testimonianze di Emilio Maggini, Viterbo, Tipolitografia Quatrini, 2009.

LEI = Pfister, Max/Schweickard, Wolfgang (dal vol. 8, 2001)/Prifti, Elton (dal vol. 15:129, 2019) (edd.), Lessico etimologico italiano, Wiesbaden, Reichert, 1979ss. 
Lepri/Vitali = Lepri, Luigi/Vitali, Daniele, Dizionario bolognese-italiano, italiano-bolognese = Dizionèri bulgnaiś-itagliàn, itagliàn-bulgnaiś, con Al rimèri dal dialàtt bulgnais̉ di Amos Lelli, Bologna, Pendragon, ${ }^{2} 2009$.

LMan = Baldini, Mauro/Totteri, Mauro, Voci dalla fiora. Lessico manzianese, Manziana, Vecchiarelli, 2007.

Lorenzetti, Luca, Nota linguistica, Nota al testo e Glossario, in: Formica, Marina/Lorenzetti, Luca (edd.), Il Misogallo romano, prefazione di Tullio De Mauro, Roma, Bulzoni, 1999, 107-199 e 667-681 (= 1999b).

Lorenzetti, Luca, Benedetto Micheli e il «Misogallo romano»: due romaneschi a confronto, Contributi di filologia dell'Italia mediana 18 (2004), 83-93.

Magro, Fabio/Soldani, Arnaldo, Il sonetto italiano. Dalle origini a oggi, Roma, Carocci, 2017. Malizia = Malizia, Giuliano, Proverbi, modi di dire e dizionario romanesco. Un prezioso vademecum per conoscere e apprezzare il linguaggio della città eterna, Roma, Newton Compton, 2002.

Mancarella $/$ Parlangèli/Salamac $=$ Mancarella, Giovan Battista $/$ Parlangèli, Paola $/$ Salamac, Pietro, Dizionario dialettale del Salento, Lecce, Grifo, 2011.

Martino/Alvaro = Martino, Giuseppe Antonio/Alvaro, Ettore, Dizionario dei dialetti della Calabria meridionale, Vibo Valentia, Qualecultura, 2010.

Marucci, Valerio, Il Misogallo romano e gli stornelli. Una messa a punto in margine a una recente edizione dei testi romani del 1793, Filologia e critica 26 (2001), 278-284.

Menarini, Alberto, Tizio, Caio e San Petronio. Vicende di nomi nel dialetto bolognese, Bologna, Tamari, 1968.

Mereghi, Paolo, 100 sonetti romaneschi, Roma, Cippitelli, 1951.

Mortillaro $=$ Mortillaro, Vincenzo, Nuovo dizionario siciliano-italiano, Palermo, Lao, ${ }^{3} 1876$ (ristampa anastatica Sala Bolognese, Forni, 1970).

Muñoz, Antonio, Sinonimi del dialetto romanesco. Novanta modi per dire «imbecille», Strenna dei romanisti 8 (1947), 272-287.

NDDC = Rohlfs, Gerhard, Nuovo dizionario dialettale della Calabria, Ravenna, Longo, 1977.

PDG = Gianfranco Lotti, Le parole della gente. Dizionario dell'italiano gergale. Dalle voci burlesche medioevali ai linguaggi contemporanei dei giovani, Milano, Mondadori, 1992.

Peresio, Giovanni Camillo, Il Maggio romanesco overo Il palio conquistato. Poema epicogiocoso nel linguaggio del volgo di Roma, Ferrara, Pomatelli, 1688.

PNor = Onorati, Giuseppe, La parlata normiciana, Norma, Edizione Domusculta, 2011.

Poggi Salani, Teresa, et al. (edd.), Parole di Firenze dal «Vocabolario del fiorentino contemporaneo», Firenze, Accademia della Crusca, 2012.

Porta, Giuseppe (ed.), Anonimo romano, Cronica, Milano, Adelphi, 1979.

Possenti, Francesco (ed.), Cento anni di poesia romanesca, 2 voll., Roma, Staderini, 1966.

Prati, Angelico, Voci di gerganti, vagabondi e malviventi studiate nell'origine e nella storia, Pisa, Cursi, 1940.

PVSub = Zaccaria Antonucci, Pina, Piccolo vocabolario sublacense, Subiaco, Edizioni ITER, 1985.

Ravaro = Ravaro, Fernando, Dizionario romanesco, introduzione di Marcello Teodonio, Roma, Newton Compton, 1994.

Reho $=$ Reho, Luigi, Dizionario etimologico del monopolitano confrontato con numerosi dialetti, 2 voll., Fasano, Schena, 2008.

REWS = Faré, Paolo, Postille italiane al «Romanisches Etymologisches Wörterbuch» di W. Meyer-Lübke comprendenti le «Postille italiane e ladine» di Carlo Salvioni, Milano, Istituto Lombardo di Scienze e Lettere, 1972. 
Rohlfs, Gerhard, Grammatica storica della lingua italiana e dei suoi dialetti, 3 voll., Torino, Einaudi, 1966-1969.

Rolandi = Rolandi, Ulderico, Aggiunte e postille, in: Chiappini, Filippo, Vocabolario romanesco, ed. Migliorini, Bruno, con aggiunte e postille di Ulderico Rolandi, Roma, Chiappini Editore, ${ }^{3} 1967,343-486$.

Rossetti, Bartolomeo (ed.), Giuseppe Berneri, Il Meo Patacca, ovvero Roma in feste ne i trionfi di Vienna, Roma, Avanzini e Torraca, 1966.

RVRM = Merlo, Clemente (ed.), Raccolta di voci romane e marchiane riprodotta secondo la stampa del 1768, Roma, Società Filologica Romana, 1932.

Sabatini, Francesco, Saggio di canti popolari romani, Roma, Tipografia Tiberina, 1878.

Sanga, Glauco, Gerghi, in: Sobrero, Alberto A. (ed.), Introduzione all'italiano contemporaneo, vol. 2: La variazione e gli usi, Roma/Bari, Laterza, 1993, 151-189.

Santini, Giulio Cesare, Poesie romanesche. Antologia, ed. Possenti, Francesco, Roma, Staderini, 1962.

TB = Tommaseo, Niccolò/Bellini, Bernardo, Dizionario della lingua italiana, 8 voll., Torino, Unione Tipografico-Editrice, 1861-1879.

Tekavčić, Pavao, Grammatica storica dell'italiano, 3 voll., Bologna, il Mulino, 1972.

$\mathrm{TLF}=$ Trésor de la langue française. Dictionnaire de la langue du XIX et du XX siècle (1789-1960), 16 voll., Paris, Éditions du CNRS/Gallimard, 1971-1994.

Trebbi, Oreste/Ungarelli, Gaspare, Costumanze e tradizioni del popolo bolognese. Con pagine musicali di canti e danze, Bologna, Zanichelli, 1932.

UDia = Gaggiotti, Giancarlo, L'Umbria e i suoi dialetti. Vocabolario, etimologie, tradizioni e ricette, Perugia, Regione Umbria, Assemblea legislativa, 2015.

Vaccaro, Giulio, «Nun c'è lingua come la romana». Voci dell'antico dialetto romanesco in Giggi Zanazzo, presentazione di Claudio Costa, Roma, il Cubo, 2010.

Vaccaro, Giulio, Posso fare un unico vocabolarione romanesco? Per un «Dizionario del romanesco letterario», il 996 10:3 (2012), 65-85.

VBel = Vaccaro, Gennaro, Vocabolario romanesco belliano e italiano-romanesco, Roma, Romana Libri Alfabeto, 1969.

VCC = Cimarra, Luigi, Vocabolario del dialetto di Civita Castellana, Castel Sant'Elia, tipografia Tecnoprint, 2010.

VCor = Chiominto, Cesare, Vocaboli, espressioni, frasi idiomatiche, località, nomi, soprannomi nel dialetto di Cori. E una appendice con ninne-nanne, filastrocche, conte, nenie, canti fanciulleschi, stornelli, strambotti, prefazione di Tullio De Mauro, Roma, Bulzoni, 2006.

VCort = Felici, Sante, Vocabolario cortonese, Arezzo, Tipografie riunite Marmorini, 1985.

VCVo = Vignoli, Carlo, Il vernacolo di Castro dei Volsci, Studj romanzi 7 (1911), 117-296 (ristampa anastatica Sala Bolognese, Forni, 1988).

VDBI = Petroselli, Francesco, Vocabolario del dialetto di Blera, Viterbo, Tipolitografia Quatrini, 2010.

$\mathrm{VDBr}=$ Orsini, Giovanni, Vocabolario del dialetto braccianese. Arricchito con proverbi, detti popolari, modi di dire, usi, costumi, tradizioni, curiosità e tanto altro, Bracciano, Tuga edizioni, 2013.

VDFR = Monfeli, Paolo, Cento gusti non si possono avere: di essere bella e di saper cantare. Vocabolario del dialetto di Fabrica di Roma, Roma, Abete grafica, 1993. 
$\mathrm{VDMa}=$ Moretti, Giovanni, Vocabolario del dialetto di Magione (Perugia), prefazione di Francesco A. Ugolini, Perugia, Istituto di Filologia romanza/Opera del Vocabolario dialettale umbro, 1973.

VDS $=$ Rohlfs, Gerhard, Vocabolario dei dialetti salentini (Terra d'Otranto), 3 voll., München, Verlag der Bayerischen Akademie der Wissenschaften, 1956-1961.

VDSp = Pasquini, Dazio, Vocabolario del dialetto spellano, ed. Ugoccioni, Nicoletta, Spello, Associazione Pro Spello/Comune di Spello, 1993.

VDTi = Sciarretta, Franco, Vocabolario del dialetto tiburtino, Tivoli, Tiburis artistica, 2011.

VEI = Prati, Angelico, Vocabolario etimologico italiano, Milano, Garzanti, 1951.

VMar = Barberini, Mario, Vocabolario maremmano, Pisa, Nistri-Lischi, 1995.

Volpe $=$ Volpe, Pietro Paolo, Vocabolario napolitano-italiano tascabile compilato sui dizionarii antichi e moderni e preceduto da brevi osservazioni grammaticali appartenenti allo stesso dialetto, Napoli, Sarracino, 1869 (ristampa anastatica Sala Bolognese, Forni, 1970).

VRDR = Galli, Vincenzo (Cencio), Vocabolario e rimario in dialetto romanesco, Roma, Edizioni Rugantino, 1982.

VS = Piccitto, Giorgio/Tropea, Giovanni/Trovato, Salvatore C. (edd.), Vocabolario siciliano, 5 voll., Catania/Palermo, Centro di Studi Filologici e Linguistici Siciliani/Opera del Vocabolario siciliano, 1977-2005.

VTF = Bruschi, Renzo, Vocabolario del dialetto del territorio di Foligno, prefazione di Francesco A. Ugolini, Perugia, Istituto di Filologia romanza/Opera del Vocabolario dialettale umbro, 1980.

VTO = Mattesini, Enzo/Ugoccioni, Nicoletta, Vocabolario del dialetto del territorio orvietano, prefazione di Ignazio Baldelli, Perugia, Istituto di Filologia romanza/Opera del Vocabolario dialettale umbro, 1992.

VTT = Ugoccioni, Nicoletta/Rinaldi, Marcello, Vocabolario del dialetto di Todi e del suo territorio, Todi, Opera del Vocabolario dialettale umbro/Amministrazione comunale di Todi, 2001.

VVit = Urbani, Ezio, Il vernacolo viterbese. Glossario viterbese-italiano, italiano-viterbese con note di grammatica e accenni di fonetica, morfologia e sintassi, Viterbo, Sette città, 1999.

VVPi = Silvestrini, Marcello, Vocabolario del dialetto della Val di Pierle, premessa di Ignazio Baldelli, Perugia, Le Edizioni Università per Stranieri, 1983.

Zamboni, Alberto, Gli anglicismi nei dialetti italiani, in: Elementi stranieri nei dialetti italiani. Atti del XIV Convegno del C.S.D.I. (Ivrea, 17-19 ottobre 1984), Pisa, Pacini, 1986-1988, vol. 1 (1986), 79-125.

Zanazzo, Giggi, Tradizioni popolari romane, vol. 2: Usi, costumi e pregiudizi del popolo di Roma, Torino, Società Tipografico-Editrice Nazionale, 1908 (ristampa anastatica Sala Bolognese, Forni, 1974).

Zanazzo, Giggi, Tradizioni popolari romane, vol. 3: Canti popolari romani, con un saggio di canti del Lazio e uno studio sulle melodie romane, con note musicali del Prof. Alessandro Parisotti, Torino, Società Tipografico-Editrice Nazionale, 1910 (ristampa anastatica Sala Bolognese, Forni, 1982).

Zanazzo, Giggi, Tradizioni popolari romane, vol. 4: Proverbi romaneschi, modi proverbiali e modi di dire, ed. Orioli, Giovanni, Roma, Staderini, ${ }^{2} 1966$.

Zanazzo, Giggi, Poesie romanesche, ed. Orioli, Giovanni, 3 voll., Roma, Avanzini e Torraca, 1968. 